\title{
Prevalence of magnetic reconnection in the near-Sun heliospheric current sheet
}

T. D. Phan ${ }^{1}$, B. Lavraud ${ }^{2,3}$, J. S. Halekas ${ }^{4}$, M. Øieroset ${ }^{1}$, J. F. Drake ${ }^{5}$, J. P. Eastwood ${ }^{6}$, M. A. Shay ${ }^{7}$, P. S. Pyakurel $^{1}$, S. D. Bale ${ }^{1,6,8}$, D. Larson ${ }^{1}$, R. Livi ${ }^{1}$, P. L. Whittlesey ${ }^{1}$, A. Rahmati ${ }^{1}$, M. Pulupa ${ }^{1}$, M. D. McManus ${ }^{1}$, J. L. Verniero ${ }^{1}$, J. W. Bonnell ${ }^{1}$, N. A. Schwadron ${ }^{9}$, M. Stevens ${ }^{10}$, A. W. Case ${ }^{10}$, J. C. Kasper ${ }^{11}$, R. J. MacDowall ${ }^{12}$, P. A. Szabo ${ }^{12}$, A. Koval ${ }^{12,13}$, K. E. Korreck ${ }^{10}$, T. Dudok de Wit ${ }^{14}$, D. Malaspina ${ }^{15}$, K. Goetz ${ }^{16}$, and P. R. Harvey ${ }^{1}$

\author{
${ }^{1}$ SSL, University of California, Berkeley, CA 94720, USA \\ e-mail: phan@ssl.berkeley.edu \\ ${ }^{2}$ Laboratoire d'Astrophysique de Bordeaux, Univ. Bordeaux, France \\ ${ }^{3}$ IRAP, Université de Toulouse, France \\ ${ }^{4}$ University of Iowa, Iowa City, IA 52242, USA \\ ${ }^{5}$ University of Maryland, College Park, MD, USA \\ 6 The Blackett Laboratory, Imperial College London, London, UK \\ ${ }^{7}$ University of Delaware, Newark, DE, USA \\ ${ }^{8}$ Physics Department, University of California, Berkeley, CA 94720-7300, USA \\ ${ }^{9}$ Department of Physics, University of New Hampshire, NH, USA \\ ${ }^{10}$ Smithsonian Astrophysical Observatory, Cambridge, MA, USA \\ ${ }_{11}$ Climate and Space Sciences and Engineering, University of Michigan, Ann Arbor, MI, USA \\ 12 NASA Goddard Space Flight Center, Greenbelt, MD, USA \\ ${ }^{13}$ University of Maryland Baltimore County, Baltimore, MD, USA \\ ${ }^{14}$ LPC2E, CNRS and University of Orléans, Orléans, France \\ 15 LASP, University of Colorado Boulder, Colorado, USA \\ ${ }^{16}$ School of Physics and Astronomy, University of Minnesota, Minneapolis, MN, USA
}

Received 6 November 2020 / Accepted 16 December 2020

\begin{abstract}
During three of its first five orbits around the Sun, Parker Solar Probe (PSP) crossed the large-scale heliospheric current sheet (HCS) multiple times and provided unprecedented detailed plasma and field observations of the near-Sun HCS. We report the common detections by PSP of reconnection exhaust signatures in the HCS at heliocentric distances of 29.5-107 solar radii during encounters 1,4 , and 5. Both sunward and antisunward-directed reconnection exhausts were observed. In the sunward reconnection exhausts, PSP detected counterstreaming strahl electrons, indicating that HCS reconnection resulted in the formation of closed magnetic field lines with both ends connected to the Sun. In the antisunward exhausts, PSP observed dropouts of strahl electrons, consistent with the reconnected HCS field lines being disconnected from the Sun. The common detection of reconnection in the HCS suggests that reconnection is almost always active in the HCS near the Sun. Furthermore, the occurrence of multiple long-duration partial crossings of the HCS suggests that HCS reconnection could produce chains of large bulges with spatial dimensions of up to several solar radii. The finding of the prevalence of reconnection in the HCS is somewhat surprising since PSP has revealed that the HCS is much thicker than the kinetic scales required for reconnection onset. The observations are also in stark contrast with the apparent absence of reconnection in most of the small-scale and much more intense current sheets encountered near perihelia, many of which are associated with "switchbacks". Thus, the PSP findings suggest that large-scale dynamics, either locally in the solar wind or within the coronal source of the HCS (at the tip of helmet streamers), plays a critical role in triggering reconnection onset.
\end{abstract}

Key words. Sun: magnetic fields - Sun: heliosphere - solar wind - Sun: flares

\section{Introduction}

Magnetic reconnection in current sheets is a universal plasma process that converts magnetic energy into plasma jetting and heating, and it is important in many laboratory, space, solar, and astrophysical contexts (e.g., Parker 1983; Priest 1984; Kronberg 2002; Yamada et al. 2010; Paschmann et al. 2013). In situ spacecraft observations have revealed the occurrence of reconnection in current sheets spanning a large range of scale sizes: from the electron-scale (a few kilometers) current sheets in Earth's turbulent magnetosheath (e.g., Phan et al. 2018) to current sheets that span millions of kilometers (or ion inertial lengths) in the solar wind (e.g., Phan et al. 2006, 2009; Gosling 2007; Gosling et al. 2007; Lavraud et al. 2009).

At $1 \mathrm{AU}$, most of the solar wind reconnection exhausts reported thus far are associated with interplanetary coronal mass ejections (ICME) or in random solar wind current sheets (e.g., Gosling et al. 2005a,b; Gosling 2007; Phan et al. 2006, 2010; Davis et al. 2006; Huttunen et al. 2008; Eriksson et al. 2009; Ruffenach et al. 2012, 2015; Lavraud et al. 2014; Mistry et al. 2015, 2017). Intriguingly, however, even though the heliospheric current sheet (HCS) is perhaps the largest and most well-defined 
current sheet in the solar wind, reconnection is rarely seen in the HCS at 1 AU (Gosling et al. 2005b). As far as we know, only three reconnecting HCSs have been reported at 1 AU (Gosling et al. 2005b, 2006; Lavraud et al. 2009). Two of the reported HCS reconnection events displayed signatures of closed field lines (with their two footpoints anchored on the Sun; Gosling et al. 2006; Lavraud et al. 2009), while the other showed evidence for magnetic disconnection from the Sun (Gosling et al. 2005b). In those reported events, signatures of separatrix layers adjacent (exterior) to the exhaust were also detected, although their complex structures and intermittent nature suggest time-varying reconnection (Lavraud et al. 2009). Gosling et al. (2005b) suggested that the HCS at 1 AU might often be too thick or magnetically too weak to reconnect locally.

More generally, in-situ observations have revealed that reconnection occurs in only a fraction of current sheets detected in the Earth's magnetosphere and in the solar wind, and the time variability of reconnection is quite different in different regions. For example, reconnection in the Earth's magnetotail is highly bursty and intermittent (e.g., Angelopoulos et al. 1994). Magnetotail observations have indicated that current sheets have to be thin enough ( $\sim$ ion inertial length scale) to trigger reconnection (e.g., Sanny et al. 1994; Runov et al. 2008). Earth's dayside magnetopause current sheet is also usually thin due to the constant compression of the solar wind against the magnetosphere, but when a spacecraft crosses the magnetopause, the probability of observing ongoing reconnection locally is only about $50 \%$, even when the magnetic shear angle across the magnetopause is large (e.g., Paschmann et al. 1986; Phan \& Paschmann 1996).

This indicates that a thin current sheet is a necessary but not sufficient condition for reconnection. Theoretical and observational studies of magnetopause and solar wind reconnection suggest that the occurrence of reconnection depends on plasma and magnetic shear (Swisdak et al. 2003, 2010; Phan et al. 2010, 2013) as well as velocity shear across the current sheet (La Belle-Hamer et al. 1995; Doss et al. 2016). When magnetopause reconnection does occur, however, the process can operate in a quasi-steady state manner if the boundary conditions are stable (Frey et al. 2003; Phan et al. 2004).

Parker Solar Probe (PSP, Fox \& McComas 2016) provides a new opportunity to study the nature of the HCS in the inner heliosphere (Szabo et al. 2020; Phan et al. 2020; Lavraud et al. 2020). During its first encounter (orbit) alone, PSP detected reconnection in several HCS crossings (Phan et al. 2020; Szabo et al. 2020), hinting that reconnection in the HCS could be more common closer to the Sun than at $1 \mathrm{AU}$. The recent observations from the subsequent PSP encounters provide an opportunity to further examine the occurrence of reconnection in the near-Sun HCS. Here we present the results of a comprehensive survey of the presence or absence of reconnection exhaust signatures in the HCS encounters by PSP during its first five orbits. Our main finding is that reconnection occurs commonly in HCS crossings close to the Sun: five out of six well-defined full HCS crossings displayed accelerated flows as well as field line topology signatures that are consistent with active reconnection in the HCS. Furthermore, a few long-duration PSP partial crossings into the HCS detected plasma jetting that lasted much of the duration of the crossings, further suggesting that reconnection in those HCS's was active much of the time.

The paper is organized as follows: Sect. 2 describes the dataset used while Sect. 3 describes event selection criteria. Section 4 discusses reconnection identification. In Sect. 5, we show complete as well as partial crossings of the HCS during encounter 4 , in addition to one event in encounter 1 . The encounter 5 HCS crossings, one of which showed no reconnection jets, are described in Sect. 6. Section 7 discusses the implications of our findings on the occurrence and structure of reconnection in the near-Sun $\mathrm{HCS}$.

\section{Dataset and instrumentation}

We studied PSP HCS crossings observed during encounters 1, 4 , and 5, at distances of 29.5-107 solar radii $\left(R_{\mathrm{S}}\right)$ from the Sun. Encounters 2 and 3 data did not show complete crossings of the HCS. During encounters 4 and 5, the spacecraft orbits were close to the HCS much of the time (e.g., Badman et al. 2021; Chen et al. 2021), resulting in multiple HCS crossings. PSP crossed the HCS fully and cleanly twice in encounter 4 and twice in encounter 5 , in addition to partially dipping into the HCS a number of times.

The magnetic field was measured by the FIELDS fluxgate magnetometer (Bale et al. 2016). The magnetic field vectors were sampled at high rates $\left(5-290\right.$ vectors $\left.\mathrm{s}^{-1}\right)$, but for uniformity of data presentation and for the comparison of current density values at various radial distances (Figs. $1 \mathrm{~b}$ and $7 \mathrm{~b}$ ), the magnetic field data presented in the present paper has been averaged to $0.2 \mathrm{~s}$.

Encounters 4 and 5 have high (sub-second) resolution ion data from the SWEAP/SPAN-ion (Livi et al. 2020) and SWEAP/SPC (Kasper et al. 2016; Case et al. 2020) instruments, capable of fully resolving the HCS and detecting reconnection signatures. During encounters 4 and 5, especially near and after perihelia, the core solar wind ions were in the SPAN-ion instrument field-of-view much of the time. This allowed for good measurements of the ion moments, including the ion temperature and temperature anisotropy, which are helpful for the analysis of reconnection events. We use the best resolution data available, which were $0.87 \mathrm{~s}$ cadence for SPAN-ions and $0.44 \mathrm{~s}$ for SPC.

To investigate field line connectivity to the Sun, we use pitch angle information of $314 \mathrm{eV}$ electrons (corresponding to solar strahl electrons) measured by the SWEAP/SPAN-electron instrument (Whittlesey et al. 2020), with a highest time resolution of $0.87 \mathrm{~s}$. We also utilize electron temperature moments computed using the methodology described by Halekas et al. (2020).

\section{Event selections}

We studied all complete HCS crossings by PSP during encounters 1, 4, and 5 (hereafter referred to as E1, E4, and E5), in addition to selected partial crossings. Tables 1 and 2 list the complete and extended partial HCS crossings described in this paper, as well as their properties. The presence or absence of reconnection is a subject of our investigation, and not a requirement in the HCS event selection. Here we discuss our methods for recognizing complete and partial HCS crossings.

\subsection{Well-defined complete HCS crossings}

Complete crossings are recognized by the radial magnetic field $B_{\mathrm{R}}$ changing from one polarity to another and reaching the asymptotic states on both sides of the current sheet, together with the concurrent (or nearly concurrent) switching between $0^{\circ}$ and $180^{\circ}$ pitch angle of suprathermal (strahl) electron fluxes of solar origin. Complete HCS crossings are typically characterized by large rotation of the field, often exceeding $150^{\circ}$. Two such crossings occurred in E1, two in E4, two in E5, and none 
Table 1. Well-defined complete HCS crossings in encounters 1, 4, and 5.

\begin{tabular}{|c|c|c|c|c|c|c|c|c|c|c|c|}
\hline Orbit & $\begin{array}{l}\text { Start time }{ }^{(a)} \\
\quad(\mathrm{UT})\end{array}$ & $\begin{array}{l}\text { Crossing } \\
\operatorname{dur}^{(b)}(\mathrm{s})\end{array}$ & $\begin{array}{c}\left|V_{\mathrm{n}}\right|^{(c)} \\
\left(\mathrm{km} \mathrm{s}^{-1}\right)\end{array}$ & $\begin{array}{l}\text { Width }^{(d)} \\
\quad(\mathrm{km})\end{array}$ & $\begin{array}{l}\text { Width }^{(e)} \\
\qquad\left(d_{\mathrm{i}}\right)\end{array}$ & $\begin{array}{l}\text { PSP to X- } \\
\text { line }{ }^{(f)} \\
\quad\left(R_{\mathrm{S}}\right)\end{array}$ & $\begin{array}{l}\text { B shear }{ }^{(g)} \\
\quad(\text { deg. })\end{array}$ & $\begin{array}{c}V_{\mathrm{R}} \\
\text { jet }^{(h)}\end{array}$ & $\begin{array}{l}\text { Current sheet lmn } \\
\text { coord in } \mathrm{RTN}^{(i)}\end{array}$ & $\begin{array}{l}\text { B closed/ } \\
\operatorname{discon}^{(j)}\end{array}$ & $\begin{array}{c}\text { Distance } \\
\text { to } \operatorname{Sun}^{(k)} \\
\left(R_{\mathrm{S}}\right) \\
\end{array}$ \\
\hline 1 & 2018-11-13/16:19:00 & 1150 & 120 & 136900 & 8220 & 0.98 & 168 & + & $\begin{array}{l}{[0.82,-0.36,0.44]} \\
{[0.18,-0.58,-0.80]} \\
{[0.54,0.74,-0.41]}\end{array}$ & discon & 62.9 \\
\hline 1 & 2018-11-23/18:27:46 & 640 & 250 & 157300 & 4820 & 1.1 & 168 & + & $\begin{array}{l}{[0.64,-0.68,0.36]} \\
{[-0.07,0.41,0.91]} \\
{[0.76,0.61,-0.21]}\end{array}$ & discon & 107.2 \\
\hline 4 & 2020-01-20/03:57:38 & 217 & 116 & 25240 & 890 & 0.18 & 162 & - & $\begin{array}{c}0.80,-0.55,-0.22] \\
{[0.51,0.83,-0.23]} \\
{[0.31,0.07,0.95]}\end{array}$ & closed & 72.5 \\
\hline 4 & 2020-02-01/04:03:46 & 290 & 42 & 12300 & 1320 & 0.09 & 167 & - & $\begin{array}{l}{[0.89,-0.42,0.17]} \\
{[-0.45,-0.86,0.24]} \\
{[0.05,-0.29,-0.96]}\end{array}$ & closed & 36.5 \\
\hline 5 & $2020-06-08 / 11: 05: 56$ & 26 & 67 & 1740 & 240 & 0.012 & 171 & + & $\begin{array}{l}{[0.85,-0.45,0.27]} \\
{[-0.24,-0.79,-0.56]} \\
{[0.47,0.41,-0.78]}\end{array}$ & discon & 29.5 \\
\hline 5 & 2020-06-08/15:40:45 & 1145 & 66 & 75300 & 9770 & N/A & 144 & none & $\begin{array}{c}{[0.82,0.50,-0.27]} \\
{[0.53,0.84,0.05]} \\
{[0.21,-0.19,0.96]}\end{array}$ & closed? & 30.1 \\
\hline
\end{tabular}

Notes. ${ }^{(a)}$ Crossing time of leading edge of HCS. ${ }^{(b)}$ Duration of HCS crossing. ${ }^{(c)}$ Average normal (n) velocity measured at the two edges of the HCS, representing the current sheet normal motion relative to PSP. ${ }^{(d)}$ Width of HCS, obtained from crossing duration and normal velocity. ${ }^{(e)}$ Width of HCS, in ion inertial length based on the average density measured at the two edges of the HCS. ${ }^{(f)}$ Estimated distance from PSP to the reconnection X-line, based on the HCS half-width and 0.1 reconnection rate assumption. ${ }^{(g)}$ Magnetic field rotation angle across the HCS. ${ }^{(h)}$ Radial velocity in HCS: Enhanced (+), reduced (-), or no jet (none) relative to external solar wind flow. ${ }^{(i)}$ Current sheet (lmn) coordinate system obtained from the minimum variance analysis of the magnetic field. ${ }^{(j)}$ Closed or disconnected magnetic field signatures based on counterstreaming or dropout of strahl electron fluxes, respectively. ${ }^{(k)} \mathrm{HCS}$ crossing distance from the Sun.

Table 2. Extended partial HCS crossings in encounters 4 and 5.

\begin{tabular}{|c|c|c|c|c|c|c|c|c|}
\hline $\begin{array}{l}\text { Start time }^{(a)} \\
\text { (UT) }\end{array}$ & $\begin{array}{c}\operatorname{Dur}^{(b)} \\
(\mathrm{h})\end{array}$ & $\begin{array}{l}\text { PSP vel in } \\
\operatorname{RTN}^{(c)} \\
\left(\mathrm{km} \mathrm{s}^{-1}\right)\end{array}$ & $\begin{array}{c}\mathrm{SW} V_{\mathrm{R}} \\
\mathrm{SC} \mathrm{frame}^{(d)} \\
\left(\mathrm{km} \mathrm{s}^{-1}\right)\end{array}$ & $\begin{array}{c}\mathrm{R} \text { dimension } \\
\text { using PSP vel } \\
\left(R_{\mathrm{S}}\right)\end{array}$ & $\begin{array}{c}\text { R dimension } \\
\text { SW vel }{ }^{(f)}\left(R_{\mathrm{S}}\right)\end{array}$ & $\begin{array}{l}V_{\mathrm{R}} \text { jet in } \\
\text { current } \\
\text { sheet }^{(g)}\end{array}$ & $\begin{array}{c}\text { B } \\
\text { closed/ } \\
\text { Discon }^{(h)}\end{array}$ & $\begin{array}{c}\text { Distance to } \\
\text { Sun }^{(i)} \\
\left(R_{\mathrm{S}}\right)\end{array}$ \\
\hline 2020-01-30/13:31:00 & 3.5 & $24.5,102.2,2.5$ & 190 & 0.44 & 3.4 & + & Discon & 30 \\
\hline 2020-01-31/19:54:00 & 3.2 & $34.2,93.1,3.7$ & 225 & 0.56 & 3.7 & + & Discon & 35 \\
\hline $2020-06-04 / 03: 56: 00$ & 2.1 & $-44.2,76.9,-5.1$ & 340 & 0.47 & 3.6 & + & Discon & 39 \\
\hline
\end{tabular}

Notes. ${ }^{(a)}$ Begin time of partial HCS crossing. ${ }^{(b)}$ Duration of partial HCS crossing. ${ }^{(c)}$ Spacecraft velocity in RTN in Sun's frame. ${ }^{(d)}$ Radial component of the solar wind velocity in spacecraft frame. ${ }^{(e)}$ Estimated blob dimension along the PSP radial trajectory assuming stationary blob. ${ }^{(f)}$ Estimated blob dimension along R assuming blob propagation with the solar wind. ${ }^{(g)}$ Radial velocity in HCS: Enhanced (+), reduced (-), or no jet (none) relative to external solar wind flow. ${ }^{(h)}$ Closed or disconnected magnetic field signatures based on counterstreaming or dropout of strahl electron fluxes, respectively. ${ }^{(i)} \mathrm{HCS}$ crossing distance from the Sun.

in E2 and E3. Although not part of HCS selection criteria, $|\mathbf{B}|$ had deep minima within the current sheet in all these events. Such events are shown in Figs. 2, 6, 7i-1 and 7m-p. In some events the field rotated fully or nearly fully, but the strahl electron flux level before and/or after the field rotation was not at the asymptotic levels. This behavior may be related to the different ways the HCS is created near the Sun (e.g., Crooker et al. 2004; Huang et al. 2016), and does not affect our ability to evaluate the presence or absence of reconnection exhaust ion jets in the HCS.

There are a few long and complicated (highly nonmonotonic) complete HCS crossings, especially in E5, that we elected not to include. This is because it is difficult to judge whether reconnection occurs or not in such events.

\subsection{Partial HCS crossings}

Partial crossings are spacecraft entering and exiting the HCS on the same side without reaching the other side of the HCS. These are recognized by $B_{\mathrm{R}}$ changes away from the baseline level that do not completely reach the other asymptotic state. Such events are shown in Figs. 4, 5, and 7e-h. There are typically a number of such partial HCS crossings near the full crossing. They have smaller magnetic field rotation than the complete crossing. 


\subsection{Distinguishing partial reconnecting HCS from magnetic field switchbacks}

In terms of the $B_{\mathrm{R}}$ behavior, partial HCS crossings that exhibit reconnection jet signatures are similar to switchbacks in that (a) $B_{\mathrm{R}}$ makes an excursion from the baseline but subsequently returns to the same baseline, and (b) the flow variations across the switchbacks are Alfvenic (e.g., Horbury et al. 2018, 2020; Bale et al. 2019; Kasper et al. 2019; Phan et al. 2020). However, there are important distinctions: (1) in switchbacks, strahl electron flux intensity typically does not change as the magnetic field rotates (e.g., Bale et al. 2019; Whittlesey et al. 2020), whereas a partial HCS crossing displays big changes in strahl fluxes in the field rotation region, for example with strahl flux dropouts in antisunward-directed exhausts. (2) $|\mathbf{B}|$ in switchbacks tends to be remarkably constant, indicating a pure rotation of the field, whereas there is a significant decrease in $|\mathbf{B}|$ in partial HCS crossings, consistent with a high-magnetic shear current sheet crossing. (3) The flow acceleration associated with switchbacks is always antisunward $\left(\Delta V_{\mathrm{R}}>0\right)$ (e.g., Horbury et al. 2018, 2020; Kasper et al. 2019; Bale et al. 2019; Phan et al. 2020), whereas flow acceleration detected in partial HCS crossings can be either sunward or antisunward directed, depending on whether the $\mathrm{X}$-line is located antisunward or sunward of the spacecraft (Phan et al. 2020).

It should be noted that there might be partial crossings of HCS that do not exhibit reconnection jet signatures. Such events would not resemble Alfvénic switchbacks because of the lack of Alfvénic accelerated flows.

\section{Coordinate system and reconnection identification}

\subsection{Current sheet coordinate system}

In reconnection studies, one often examines the data in the current sheet coordinate system, although reconnection exhausts can be recognized in any coordinate systems. However, because the near-Sun HCS lies approximately in the R-T plane, for simplicity we display all data in the RTN coordinate system (see Fig. 3), with the $\mathrm{N}$ direction perpendicular to the ecliptic plane, which is often close to the HCS plane, especially close to the Sun. Furthermore, the velocity data shown in this paper is in the Sun's frame. However, for the estimation of the HCS thickness, we use the velocity data in the spacecraft frame and in the current sheet (lmn) coordinate system obtained using the minimum variance analysis of the magnetic field (MVAB; Sonnerup \& Cahill 1967), which is found to work well for large magnetic shear current sheets such as the HCS. The lmn coordinates of the complete HCS crossings are given in Table 1. For HCS crossings close to the Sun (in E4 and E5), the current sheet lmn coordinates are close to RTN, with $1 \sim \mathrm{R}, \mathrm{m} \sim \mathrm{T}$, and $\mathrm{n} \sim \mathrm{N}$.

\subsection{Reconnection and non-reconnection event identification}

We identify reconnection exhausts in current sheets by the presence of ion jets with respect to the ambient solar wind frame, which as an example may correspond to accelerated (increase in $V_{\mathrm{R}}$ ) or decelerated (decrease in $V_{\mathrm{R}}$ ) radial ion flows in the exhaust for exhausts aligned with the radial direction (but note that the jets may have significant components in other directions as well). These flows are bounded on one edge of the current sheet by correlated changes in velocity, $\mathbf{V}$, and magnetic field, $\mathbf{B}$, and anti-correlated changes in $\mathbf{V}$ and $\mathbf{B}$ on the other edge, indicating a pair of rotational discontinuities (RDs) bounding the exhaust. Such opposite correlations between the changes in $\mathbf{V}$ and $\mathbf{B}$ are consistent with Alfvénic disturbances propagating in opposite directions along reconnected field lines away from the reconnection site (X-line; e.g., Gosling et al. 2005a). We do not identify reconnection events based on magnetic field profiles such as deep minima in $|\mathbf{B}|$ or bifurcated current sheets alone, as they are not unique to reconnection events.

In contrast, non-reconnecting current sheets are recognized by the absence of plasma jetting within the current sheets. In such cases, the current sheet could be a tangential discontinuity (TD) or a single rotational discontinuity (RD). Across a TD, the $V$ and $B$ variations are usually not correlated since the plasmas on the two sides of a TD are not magnetically connected with each other. Across a single RD, there should be a single correlation between $\mathbf{V}$ and $\mathbf{B}$. An example of a current sheet with no reconnection jetting is shown in Figs. $7 \mathrm{~m}-\mathrm{p}$.

For complete HCS crossings, the above qualitative way of identifying reconnection events is usually sufficient. For partial crossings, however, since the spacecraft does not sample both edges of the HCS, we examine the flows inside the HCS more quantitatively by comparing the observed flows to the Walén relation for a rotational discontinuity (e.g., Hudson 1970; Sonnerup et al. 1981; Paschmann et al. 1986). Because SWEAP/SPAN-ion and SPAN-electron provides reliable temperature measurements, we use the most general form of the Walén relation, which takes into account the plasma pressure anisotropy:

$$
\begin{aligned}
\Delta \mathbf{V}_{\text {predicted }} & =\mathbf{V}_{2}-\mathbf{V}_{1} \\
& = \pm \mathbf{B}_{2}\left(1-\alpha_{2}\right)^{1 / 2}\left(\mu_{0} \rho_{2}\right)^{-1 / 2}-\mathbf{B}_{1}\left(1-\alpha_{1}\right)^{1 / 2}\left(\mu_{0} \rho_{1}\right)^{-1 / 2} .
\end{aligned}
$$

Subscript ' 1 ' denotes the reference time and ' 2 ' denotes the prediction for all other times. The pressure anisotropy factor is $\alpha=\left(\mathrm{p}_{\|}-\mathrm{p}_{\perp}\right) \mu_{0} / B^{2}$, where $\mathrm{p}_{\|}$and $\mathrm{p}_{\perp}$ are the plasma (ion+electron) pressures parallel and perpendicular to $\mathbf{B}$. The ' + ' or '-' sign is used depending on whether the observed flow change across the current sheet boundary is correlated or anticorrelated with the magnetic field change. Note that the predicted flows are for a rotational discontinuity (RD), which tends to overestimate the flow acceleration in symmetric reconnection exhausts, because the exhaust boundaries in symmetric reconnection are generally a combination of slow shock (SS) and $\mathrm{RD}$, or mainly SS in the case of antiparallel (zero guide field) reconnection. Past observations and simulations have indicated that exhaust flow speed in symmetric reconnection could be as low as $40-50 \%$ of the Walén prediction (Sonnerup et al. 1981; Paschmann et al. 1986; Øieroset et al. 2000; Liu et al. 2012; Haggerty et al. 2018).

\section{Encounter 4}

\subsection{Overview}

Figure 1 shows the large-scale context of the PSP encounter 4 observations of the solar wind from 2020 January 20 to 2020 February 1, with magnetic field magnitude (Panel a) increasing with decreasing distance to the Sun, reaching $\sim 150$ nT near perihelion. Large fluctuations in the magnetic field (Panel c) are seen throughout the encounter. Figure $1 \mathrm{~b}$ reveals these fluctuations to be associated with current density spikes, reaching $|\mathbf{j}| \sim$ $1.2 \mu \mathrm{A} \mathrm{m}^{-2}$ near perihelion on January 28-29. Most of the large current density spikes are associated with magnetic switchbacks. 


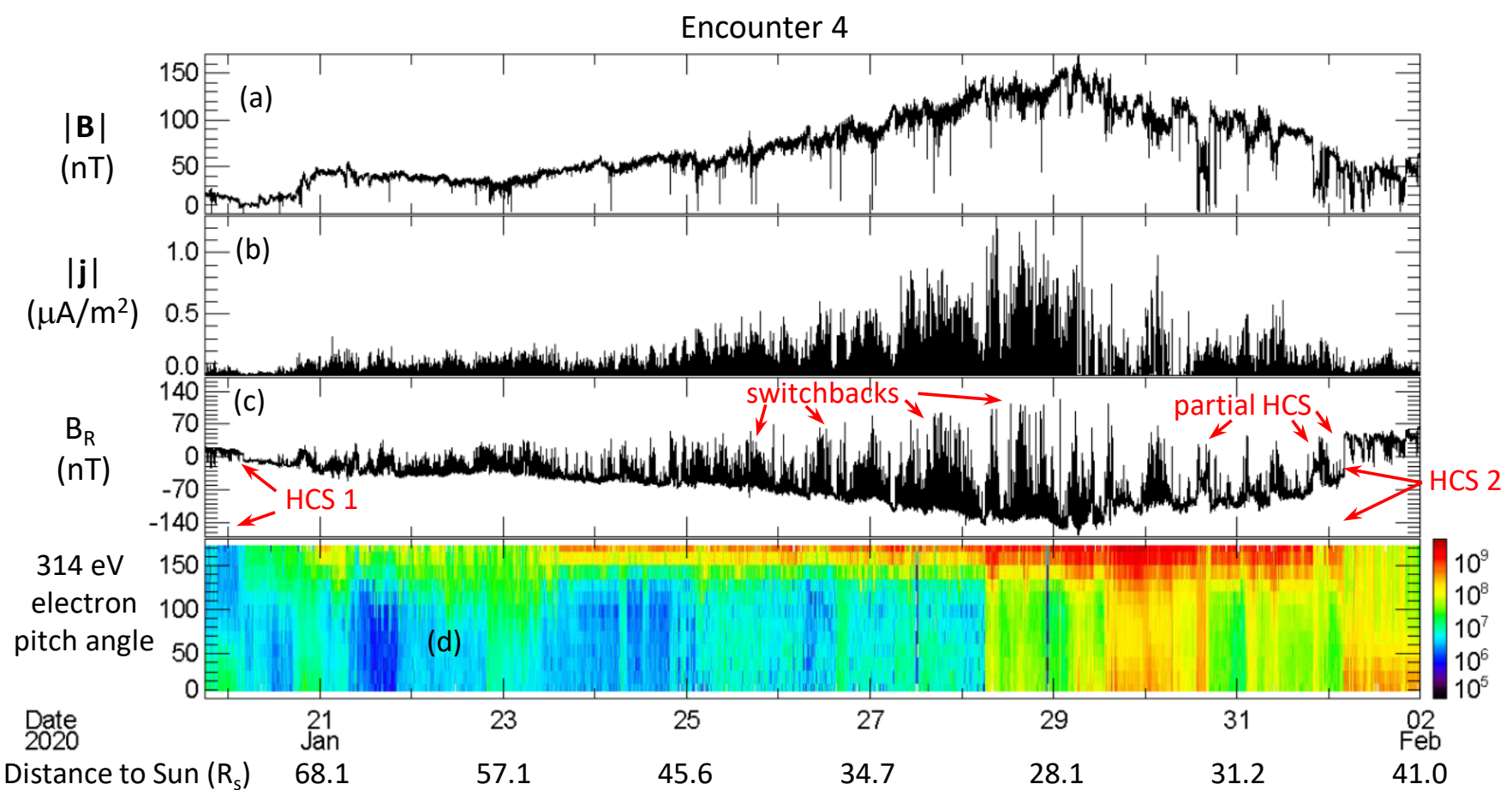

Fig. 1. Overview of PSP encounter 4 showing the locations of the heliospheric current sheets discussed in Figs. 2, 4-6. (a) Magnetic field magnitude, $(b)$ proxy for current density: $\left.|\mathbf{j}|=\left[\left(\delta B_{\mathrm{R}} / \delta \mathrm{t}\right)^{2}+\left(\delta B_{\mathrm{T}} / \delta \mathrm{t}\right)^{2}+\left(\delta B_{\mathrm{N}} / \delta \mathrm{t}\right)^{2}\right)\right]^{1 / 2} /\left(\mu_{0} V_{\mathrm{pR}}\right)$, where $V_{\mathrm{pR}}$ is the radial component of the proton velocity, $(c)$ radial component of the magnetic field, $(d)$ pitch angle energy distributions of $314 \mathrm{eV}$ (strahl) electrons measured by SWEAP/SPAN-electrons, in unit of energy flux $\left(\mathrm{eV} \mathrm{s}^{-1} \mathrm{~cm}^{-2} \mathrm{ster}^{-1} \mathrm{eV}^{-1}\right)$.

During E4, there were two well-defined complete HCS crossings (labeled 'HCS 1' and 'HCS 2' in Fig. 1c), marked by polarity reversals of the large-scale radial magnetic field, together with the concurrent switching between $0^{\circ}$ and $180^{\circ}$ pitch angle of fluxes of $314 \mathrm{eV}$ electrons, which are strahl electrons of solar origin. The two complete HCS crossings are presented in detail in the next sections, starting with HCS2 which had the best coverage in terms of high resolution data. There were also numerous partial crossings of the HCS. The ones that are discussed in details in the next sections are marked in Fig. 1c.

In the next 3 sections, we describe a sequence of HCS crossings, which together reveal a possible picture of the structure and evolution of a reconnecting HCS. We first examine the HCS 2 full crossing, and proceed backward in time to a series of partial crossings.

\subsection{HCS 2: Sunward exhaust and the formation of closed field lines}

Figure 2 shows a complete crossing of the HCS on 2020 February 1 , labeled 'HCS 2' in Fig. 1c, with polarity change of $B_{\mathrm{R}}$, which is the main magnetic field component bounding the HCS (Fig. 2b) and concurrent switching of strahl electron pitch angle fluxes from $180^{\circ}$ to $0^{\circ}$ (Fig. $2 \mathrm{~h}$ ) across the current sheet. The crossing occurred at $\sim 36.5 R_{\mathrm{S}}$ from the Sun. The magnetic field rotation across the current sheet was $167^{\circ}$, that is to say the magnetic fields on the two sides of the current sheet were nearly antiparallel. There was a deep minimum in $|\mathbf{B}|$ in the current sheet (Fig. 2a). The current sheet was bifurcated, with sharp changes in $B_{\mathrm{R}}$ at the two current sheet edges (Figs. 2b and 3). Embedded in the current sheet is a proton jet in the radial direction $\Delta V_{\mathrm{R}} \sim-30 \mathrm{~km} \mathrm{~s}^{-1}$ (relative to the external flows), which is the dominant component of the flow acceleration in this case, with opposite $\delta V_{\mathrm{pR}}-\delta B_{\mathrm{R}}$ correlations at the left and right sides of the current sheet, consistent with reconnection. The magnitude of the jet is $\sim 55 \%$ of the predicted flow change based on the Walén relation (see Sect. 4.2). The sub-Alfvénic flow speed is common for solar wind reconnection exhausts and in models of symmetric reconnection (e.g., Liu et al. 2012; Haggerty et al. 2018). The negative $\Delta V_{\mathrm{R}}$ jet implies that this is a sunwarddirected exhaust, that is to say the X-line was located antisunward of PSP (see Fig. 3). Also consistent with reconnection are density (Fig. 2d) and temperature (Fig. 2e) enhancements inside the current sheet (e.g., Gosling et al. 2005a), with $\Delta T_{\mathrm{p} \|}$ greater than $\Delta T_{\mathrm{p} \perp}$ (e.g., Gosling et al. 2005a; Phan et al. 2014) as shown in Fig. $2 \mathrm{f}$. The average ion (total) temperature increase, that is to say the difference between the average exhaust temperature $(22.3 \mathrm{eV})$ and external temperature $(20.80 \mathrm{eV})$, was only $1.5 \mathrm{eV}$. However, this is consistent with the expectation from the scaling of reconnection ion heating with the available magnetic energy found in previous solar wind and magnetopause reconnection studies: $\Delta T_{\mathrm{i}}=0.13 m_{\mathrm{i}} V_{\mathrm{A}}^{2}$ (Drake et al. 2009; Phan et al. 2014; Haggerty et al. 2015). For this event, $V_{\mathrm{A}} \sim 36 \mathrm{~km}$ (based on $\mathrm{B} \sim$ $40 \mathrm{nT}$ and density $\sim 600 \mathrm{~cm}^{-3}$ ), thus the predicted ion heating is $1.6 \mathrm{eV}$. Figure $2 \mathrm{~g}$ shows no evidence for electron heating. However, the predicted electron heating $\left(\Delta T_{\mathrm{e}}=0.017 m_{i} V_{\mathrm{A}}^{2}\right.$ (Phan et al. 2013; Shay et al. 2014)) is only $0.25 \mathrm{eV}$, and therefore would not be discernable in the data.

The duration of the HCS crossing was 290 s, which translates to an exhaust width (along the current sheet normal) of $1.2 \times 10^{4} \mathrm{~km}$, or $\sim 1320$ ion inertial lengths $\left(d_{\mathrm{i}}\right)$, based on the measured normal velocity of the current sheet relative to the spacecraft of $\sim 42 \mathrm{~km} \mathrm{~s}^{-1}$ (see Table 1). If one assumes a canonical reconnection rate of 0.1 , the estimated distance from the spacecraft to the X-line is $6 \times 10^{4} \mathrm{~km}\left(6500 d_{\mathrm{i}}\right)$, or $\sim 0.09 R_{\mathrm{S}}$. The durations of the sharp $\left(B_{\mathrm{R}}\right)$ exhaust boundary crossings were 


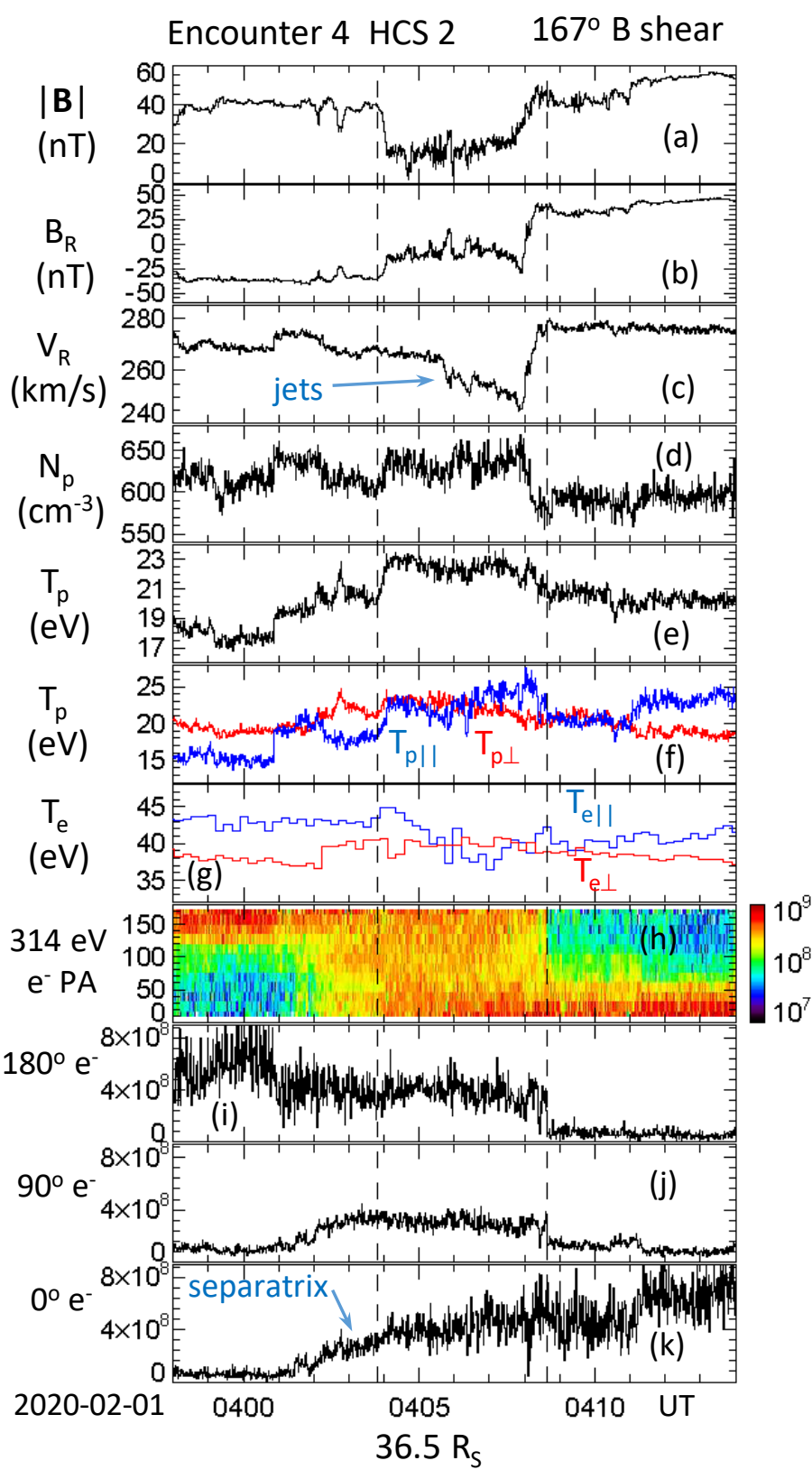

Fig. 2. Zoom-in of Fig. 1 displaying a sunward-directed reconnection exhaust and the formation of closed field lines at HCS 2. The proton moments are from the SWEAP/SPAN-ion measurements and in the Sun's frame. $(a, b)$ Magnitude and radial component of the magnetic field, $(c)$ radial component of the proton velocity, $(d)$ proton density, $(e)$ total proton temperature, $(f)$ parallel and perpendicular proton temperatures, $(g)$ electron temperature, $(h)$ pitch angle distribution of strahl electrons, $(i-k)$ differential energy fluxes $\left(\mathrm{eV} \mathrm{s}^{-1} \mathrm{~cm}^{-2} \mathrm{ster}^{-1} \mathrm{eV}^{-1}\right)$ of $314 \mathrm{eV}$ (strahl) electrons at $0^{\circ}-30^{\circ}, 75^{\circ}-105^{\circ}$, and $150^{\circ}-180^{\circ}$ pitch angles. The vertical dashed lines mark the edges of the exhaust based and magnetic field and plasma variations.

8s on the left and $26 \mathrm{~s}$ on the right, which correspond to $\sim 335 \mathrm{~km}$ $\left(37 d_{\mathrm{i}}\right)$ and $1090 \mathrm{~km}\left(120 d_{\mathrm{i}}\right)$, respectively.

The pitch angle behavior of strahl electrons can give an indication of the magnetic field line topology of the HCS (e.g., Gosling et al. 2005b, 2006; Lavraud et al. 2009, 2020). Antifield-aligned $\left(180^{\circ}\right)$ strahl electrons on sunward pointing field $\left(B_{\mathrm{R}}<0\right)$ and field-aligned $\left(0^{\circ}\right)$ electrons on antisunwardpointing field $\left(B_{\mathrm{R}}>0\right)$ were present on the two sides of the current sheet, respectively, and both are indicative of open field lines with one end connected to the Sun. Inside the current sheet (between the two vertical dashed lines in Fig. 2) both electron populations were observed (in addition to flux enhancements at intermediate pitch angles; Fig. $2 \mathrm{~h}$ ). The counterstreaming electrons at $0^{\circ}$ and $180^{\circ}$ pitch angle inside the current sheet indicate the formation of closed field lines due to reconnection at a reconnection site antisunward of PSP (see Fig. 3; e.g., Gosling et al. 2006; Lavraud et al. 2009).

A separatrix layer outside the current sheet was also observed, but only on the left edge of the current sheet. The $0^{\circ}$ $30^{\circ}$ electrons, which originated from the right side of the HCS, went through the left edge of the current sheet (Figs. 2h,k) and populated a layer outside the current sheet. The separatrix layer is less apparent on the right side, as the $150^{\circ}-180^{\circ}$ flux dropped sharply just outside the right edge of the current sheet. Such a left-right asymmetry in the duration of the separatrix layer had also been seen at HCS at 1 AU (e.g., Gosling et al. 2006; Lavraud et al. 2009). Enhancements of electron fluxes at intermediate pitch angles are also seen in the left separatrix layer, with a local peak at $90^{\circ}$. The enhanced electron fluxes at intermediate pitch angles in the current sheet may indicate scattering by waves. However, scattering probably cannot account for the $90^{\circ}$ electron flux enhancement in the left separatrix layer.

\subsection{Brief partial HCS crossings near HCS 2}

Going back in time from HCS 2, Fig. 4 shows that at $1-2 \mathrm{~h}$ before the HCS 2 crossing, PSP made three distinct partial crossings into and out of the HCS. The partial crossings are recognized by the brief $B_{\mathrm{R}}$ excursion from the negative baseline toward zero, but without actually changing polarity. We now discuss the plasma signatures of these partial crossings in comparison with those observed in HCS 2 described above.

During the three partial crossings, PSP detected antisunward $\left(\Delta V_{\mathrm{R}}>0\right)$ jets (relative to the outside flow; Fig. 4c), which is opposite to the sunward $\left(\Delta V_{\mathrm{R}}<0\right)$ jets seen in HCS 2 (Fig. 2c). Note that because PSP only crossed one side $\left(B_{\mathrm{R}}<0\right)$ of the current sheet, only the positive sign of the Walén relation was used in the prediction (red traces in Fig. 4c). The observed jet speed agrees with the Walén prediction rather well. Furthermore, PSP observed dropouts of $150^{\circ}-180^{\circ}$ strahl electron fluxes during the three partial crossings (Figs. 4h,i), as opposed to the counterstreaming $0^{\circ}$ and $180^{\circ}$ strahl electrons seen in HCS2 (Fig. 2). The antisunward jet and the electron dropouts are both consistent with the presence of a reconnection X-line located sunward of PSP, causing the reconnected field lines to be disconnected from the Sun (Gosling et al. 2005b; Lavraud et al. 2020). Separatrix signatures, with strahl dropouts starting outside the current sheet, are seen adjacent to some of the current sheet edges (Figs. 4h,i), but the durations of the separatrix layer crossings are different for different crossings, and display left-right (inbound-outbound) asymmetries.

PSP detected weak (1-2 eV) ion heating in all three partial crossings. However, in contrast to the dominantly parallel ion heating seen in HCS 2 (Fig. 2f), $T_{\mathrm{p} \|}$ actually decreased inside the current sheet, while $T_{\mathrm{p} \perp}$ increased (Fig. 4f). As far as we know, such parallel temperature behavior has not been observed in reconnection exhausts, which usually displays parallel heating due to counterstreaming ion beams (Gosling et al. 2005a; Haggerty et al. 2015). We suspect that the unexpected temperature behavior in this event may be due to the large temperature anisotropy $\left(T_{\mathrm{p} \|} \gg T_{\mathrm{p} \perp}\right)$ outside the current sheet (Fig. 4f) which could render the exhaust firehose unstable. Such a boundary condition has not been observed or simulated before. 


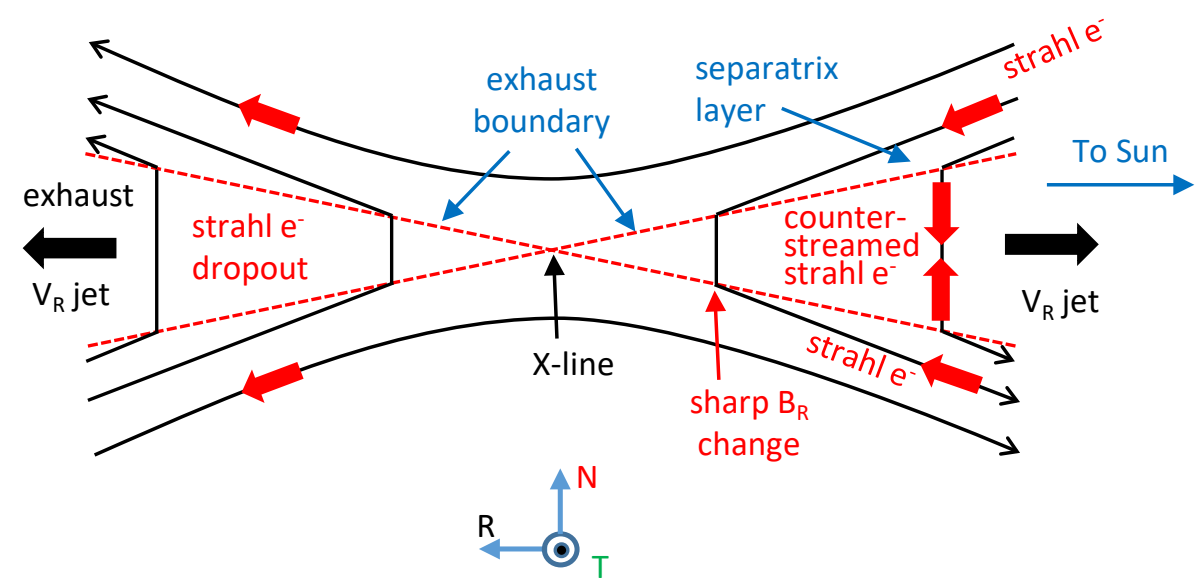

Fig. 3. Schematic illustration of the reconnection configuration and the RTN coordinate system. The (antisunward) exhaust to the left of the X-line is magnetically disconnected from the Sun. Field lines in the sunward exhaust are connected both ends to the Sun.
The large field-aligned anisotropy seems to persist for days in the solar wind close to the HCS (see Fig. 5), but interestingly, the anisotropy changed as HCS 2 was approached (Fig. 2f).

\subsection{Long duration partial HCS crossings and magnetic disconnection}

Going even further back in time, Fig. 5 shows a one-day interval preceding HCS 2 and the brief HCS crossings just described In this long interval, two periods (labeled 'partial 4' and 'partial 5'), each lasting more than three hours, show deep minima in $|\mathbf{B}|$ (Fig. 5a), and $B_{\mathrm{R}}$ turning toward a positive orientation (Fig. 5b). The partial 4 interval shows positive enhancements of $V_{R}$ (Fig. 5c) and $150^{\circ}-180^{\circ}$ strahl electron flux dropouts (Figs. 5h,i), similar to the brief partial HCS crossings described in the last section. On the other hand, partial 5 also shows positive enhancements of $V_{R}$, but strahl electron flux dropouts only occurred near the right boundary of the crossing (Figs. 5h,i). The rest of the interval shows intermittent appearance of the $150^{\circ}-180^{\circ}$ strahl electrons, as well as enhancements of fluxes at intermediate pitch angles (Fig. 5h). Thus, the magnetic topology of partial 5 is rather complex, displaying a mixture of connected and disconnected field lines (from the Sun). The intermediate flux enhancements were also seen in the partial 3 crossing (Fig. 4h), and is commonly seen in strahl dropout events in the solar wind (e.g., Crooker et al. 2003; Pagel et al. 2005; Halekas et al. 2021). Wave scattering is a possible source of the electron isotropization in these weak magnetic field, high $\beta$ regions (e.g., Crooker et al. 2003; Pagel et al. 2005; Cattell et al. 2021, 2020). The complexity in electron strahl data and Walén relations within the HCS is similar to previous HCS observations by Szabo et al. (2020) and Lavraud et al. (2020).

In both partials 4 and 5, PSP observed reduced ion parallel temperature and enhanced $T_{\mathrm{p} \perp}$ inside this interval, relative to outside. This feature is qualitatively similar to the brief (2-3 min duration) partial HCS crossings in Fig. 4. The many similarities suggest that these long duration structures (partials 4 and 5) also represent partial crossings of an antisunward exhaust. The big difference is that these two intervals are three hours long, which would suggest a very thick (along N) and long (along R) exhaust.

Figure $5 \mathrm{c}$ shows that the $V_{\mathrm{R}}$ flow enhancements in partial 4 agree reasonably well with the Walén prediction (red trace) based on the local conditions outside the current sheet. This suggests that the plasma was accelerated across the local current sheet boundaries. The fact that the accelerated flows were observed throughout the 3-h (partial) crossing of the HCS suggests that reconnection was active for at least that long. The observed $V_{\mathrm{R}}$ flows in partial 5, on the other hand, do not agree with the Walén prediction as well, especially across the right edge of the crossing. The observed $V_{\mathrm{R}}$ increase of $\sim 100 \mathrm{~km} \mathrm{~s}^{-1}$ entering the current sheet from the right side is markedly higher than predicted based on the condition just outside (to the right of) the current sheet. A reconnection jet speed that is higher than predicted is rarely seen in reconnection exhausts (Paschmann et al. 1986; Phan et al. 1996). This seems to imply that, even though the observed accelerated flows were suggestive of reconnection exhaust jets, the plasma was not accelerated across the local boundary.

Finally, we note that the magnetic field, flow acceleration, and electron properties of the HCS crossings described here closely resemble those of the encounter 1 heliospheric plasma sheet (HPS) reported by Lavraud et al. (2020). Because the main density enhancements in the vicinity of HCS crossings were observed inside (and likely produced by) the reconnection exhausts, Lavraud et al. (2020) suggested that the HPS, defined as a density enhancement close to the HCS, often solely consists of a dense and broad HCS reconnection exhaust.

\subsection{Global view of HCS 2 and prior partial crossings}

We now attempt to reconstruct a possible big picture of the structure and evolution of the HCS reconnection that ties together the observations of the multiple partial crossings and subsequently a full crossing of HCS 2. A possible scenario is depicted in Fig. 5j. In this scenario, a dominant X-line was first located sunward of PSP, producing antisunward jets observed by PSP during the multiple partial crossings. During these times, the HCS magnetic field lines were disconnected from the Sun due to sunward-of-PSP reconnection. The dominant X-line then likely moved antisunward, because by the time PSP crossed the 'thin' HCS 2, the X-line was antisunward of PSP, producing sunward jets. As PSP was in the sunward-directed exhaust, it observed closed HCS field lines with both ends connected to the Sun.

The multiple extended and brief partial HCS crossings by PSP indicate that the crossings were not caused by the wavy (flapping) motion of a uniformly thin HCS, because in such a scenario, one would have expected the spacecraft to cross the current sheet completely from time to time, instead of repeatedly entering and exiting on the same side of the current sheet. The more likely scenario is that the multiple partial crossings were caused by traveling large plasma blobs bulging onto both sides of the current sheet (e.g., Phan et al. 2004). Furthermore, since the observed flow acceleration $\Delta V_{\mathrm{R}}$ was always positive from one partial crossing to the next, or during the hours PSP stayed in 


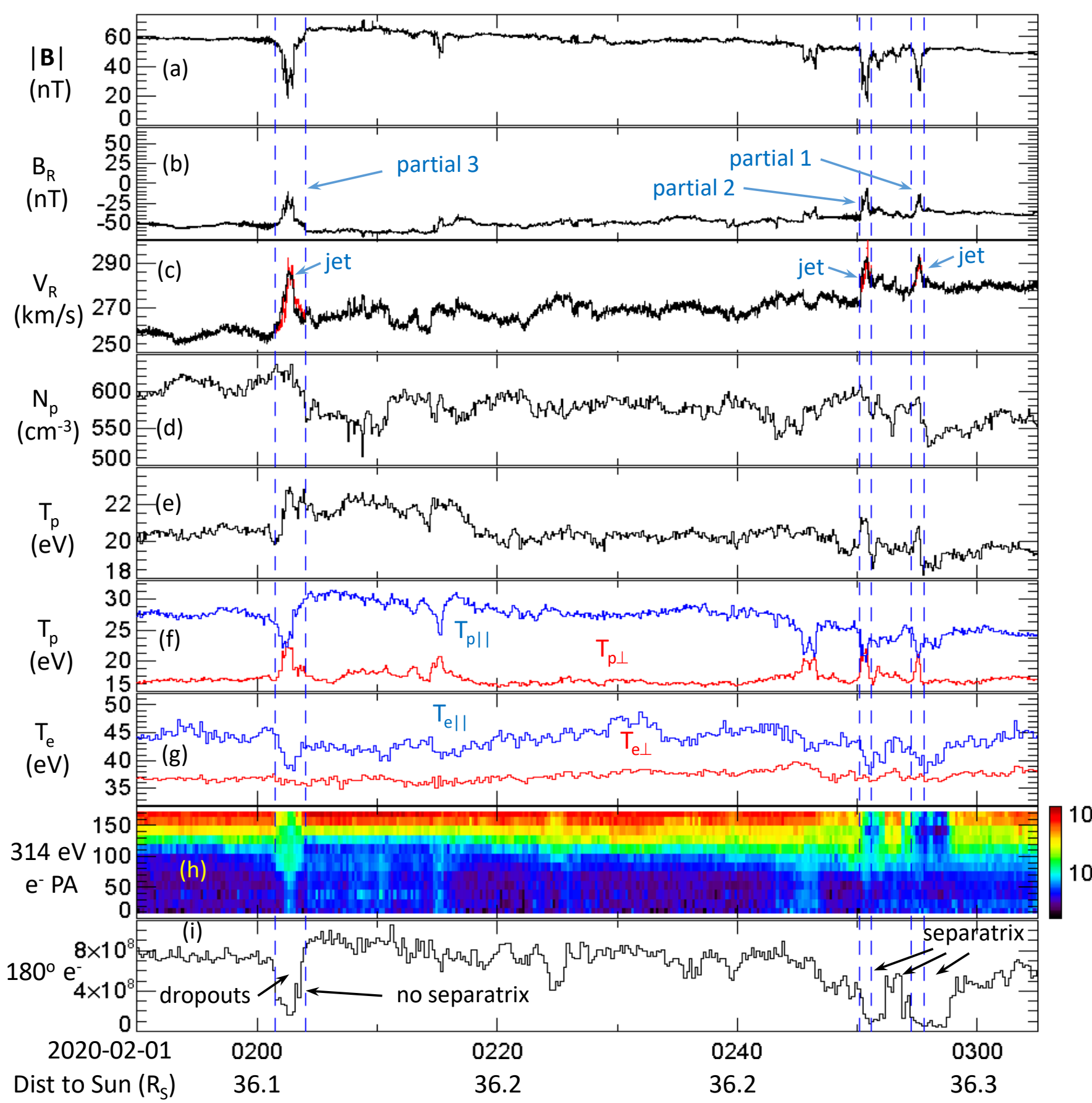

Fig. 4. Zoom-in of Fig. 1 showing three brief partial crossings of the HCS just before full HCS 2 crossing. The parameters are the same as in Fig. 2. In panel $c$, the red traces are the Walén predicted $V_{\mathrm{R}}$, computed starting from both left and right edges of each partial crossing, and ending in the middle. The three partial crossings detected enhanced $V_{R}$ (antisunward) jets and strahl electron dropouts indicative of magnetic disconnection from the Sun. Separatrix layers were clearly observed adjacent to partial crossing 1, only on the right side of partial crossing 2 , and not apparent at partial crossing 3 . The blue vertical dashed lines mark the edges of current sheet, where $B_{\mathrm{R}}$ starts to deviate from its asymptotic value.

the HCS during partials 4 and 5, it indicates that the blobs were not flanked by multiple active dominant X-lines, which would have produced alternating sunward and antisunward jets (in the solar wind frame) inside the exhaust. This scenario is similar with those proposed by Sanchez-Diaz et al. (2019) and Lavraud et al. (2020), but emphasizing that the dominant reconnection X-line may be propagating outward with the solar wind, thus sometimes forming closed field lines even far out in the solar wind.
One could estimate the minimum radial dimension of the blobs with assumptions on the relative motion of blobs relative to the spacecraft, which may represent the lower and upper limits of the motion of the blobs. First, one could assume the blobs to be stationary in the Sun's frame and use the spacecraft velocity to give the blob dimension along the spacecraft path (which is likely not the full $\mathrm{R}$ dimension of the blobs). Another assumption could be that the blobs convect with the same speed as the external solar wind flows, a scenario that is somewhat similar 

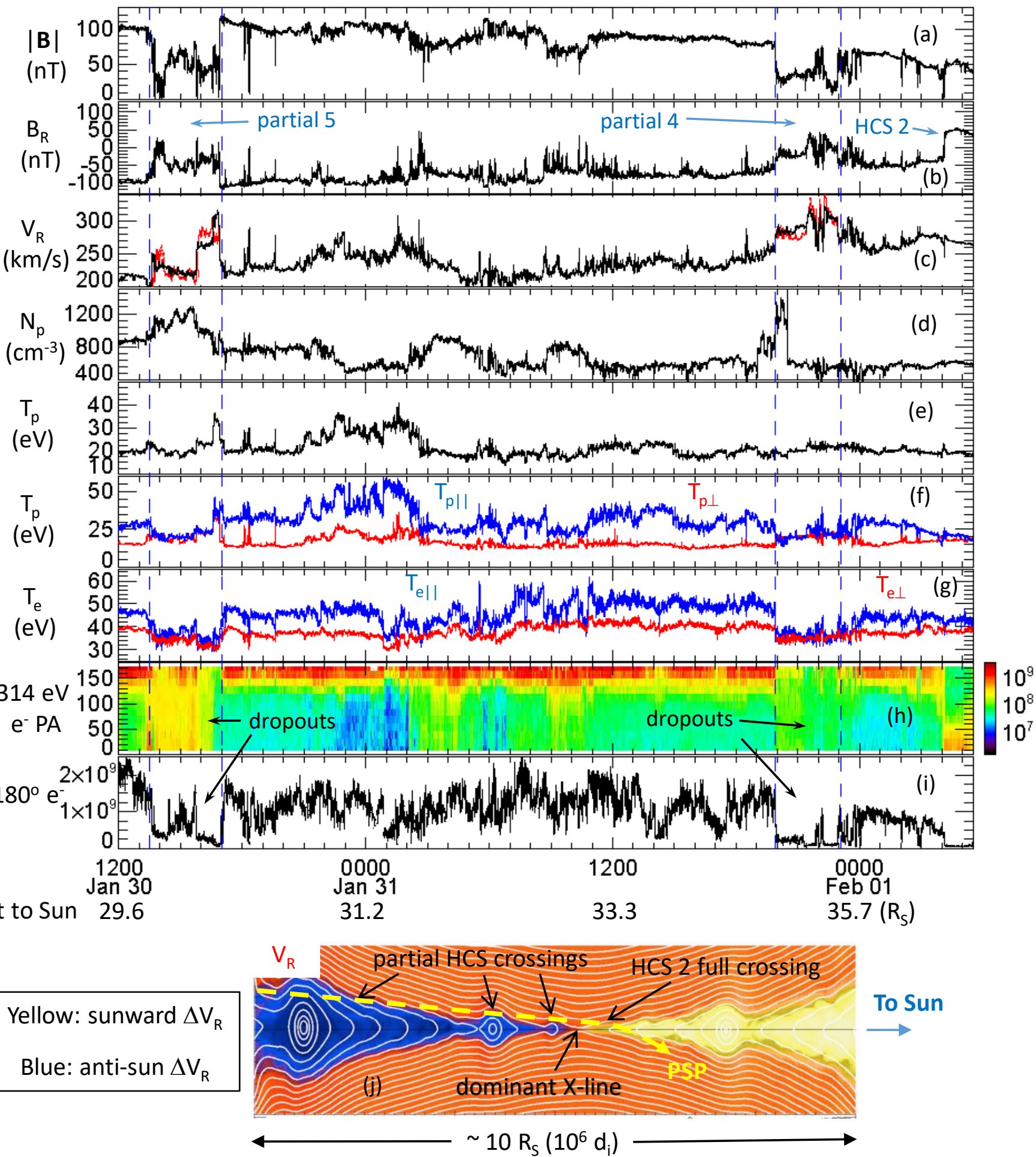

To Sun

Yellow: sunward $\Delta \mathrm{V}_{\mathrm{R}}$

Blue: anti-sun $\Delta \mathrm{V}_{\mathrm{R}}$

Fig. 5. Zoom-in of Fig. 1 showing two extended partial crossings of the HCS before the complete crossing at HCS 2. The parameters in panels a- $i$ are the same as in Fig. 2. In panel $c$, the red traces are the Walén predicted $V_{R}$, computed starting from both left and right edges of each partial crossing, and ending in the middle. The magnetic structures shown in panel $j$ is adapted from Daughton \& Karimabadi (2007) kinetic simulation of reconnection, and serve as a sketch to illustrate the effective trajectory of PSP skimming and partially penetrating into thick and blob-like structures in an antisunward exhaust, before fully crossing a thin sunward-directed exhaust on the other side of a dominant X-line. The simulation domain is three orders of magnitude smaller than the PSP context.

to how magnetic flux ropes at Earth's magnetopause move with the shocked solar wind. The latter assumption is probably more realistic, but the former assumption provides a lower limit. The estimated sizes of the partials 4 and 5 structures using these two assumptions are shown in Table 2. The lower and upper limits of the blob radial dimensions are $\sim 0.5 R_{\mathrm{S}}$ and $\sim 3.5 R_{\mathrm{S}}$. 

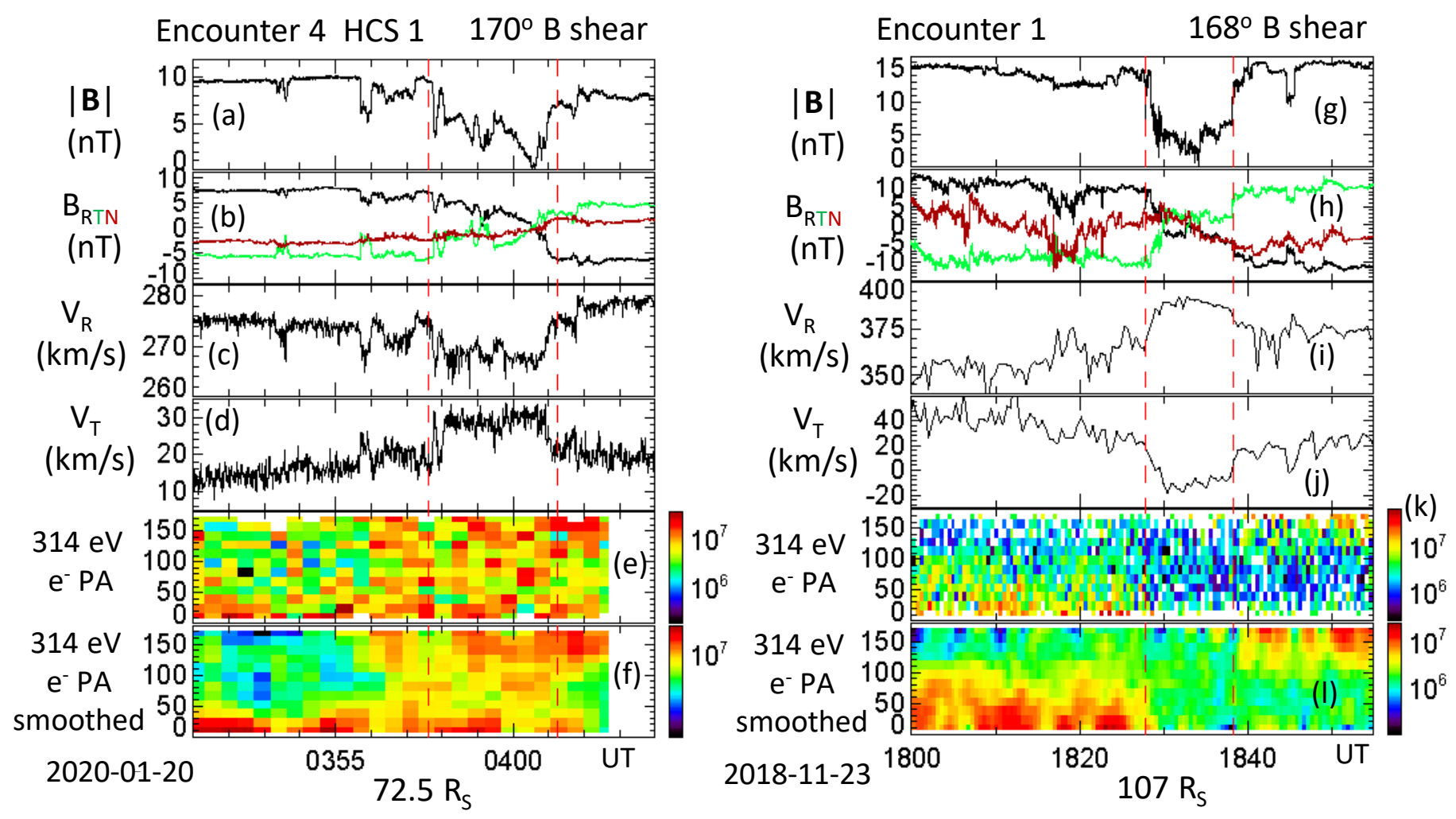

Fig. 6. Sunward-directed exhaust showing signatures of closed magnetic field lines with both ends on the Sun (left column), and an antisunward exhaust showing signatures of field lines disconnected from the Sun (right column). Panels $a$, $g$ : magnitude of the magnetic field. Panels $b$, $h$ : RTN components of the magnetic field. Panels $c, i$ : radial component of the proton velocity. Panels $d, j$ : T component of the proton velocity. Panels e-k: pitch angle distributions of strahl electrons. Panels $f, l$ : smoothed data with a running average window of $60 \mathrm{~s}$ in time and $30^{\circ}$ in pitch angle. The proton moments are from the SWEAP/SPC measurements and in the Sun's frame.

\subsection{Encounter 4 HCS 1 and encounter 1 HCS: Sunward and antisunward exhausts}

In this section we show two additional well-defined complete crossings of the HCS, with large magnetic shears and concurrent switching between $0^{\circ}$ and $180^{\circ}$ strahl electron fluxes. Both events were detected relatively far from the Sun $\left(72.5 R_{\mathrm{S}}\right.$ and $107 R_{\mathrm{S}}$ ). In one event, PSP was in a sunward-directed exhaust and observed evidence for closed magnetic field lines. In the other event, PSP was in an antisunward exhaust and detected evidence for magnetic disconnection from the Sun.

The left column of Fig. 6 shows an HCS crossing on 2020 January 20 that occurred during the inbound leg of encounter 4, labeled 'HCS 1' in Fig. 1c. Both $B_{\mathrm{R}}$ and $B_{\mathrm{T}}$ reversed sign (panel b), and the total magnetic shear across the current sheet was $170^{\circ}$, that is to say there was essentially no guide field. Plasma jetting was seen in both the $\mathrm{R}$ (panel c) and $\mathrm{T}$ (panel d) components of the ion velocity. The jet resulted in a reduction of $V_{\mathrm{R}}$, indicating that PSP was in a sunward-directed exhaust, with the X-line located antisunward of PSP. To investigate the magnetic connection to the Sun, we examined the pitch angle information of the strahl electrons. Figure 6e shows the raw highresolution (burst mode) data, which looks noisy because of the low flux intensity of strahl electrons far from the Sun. Figure $6 f$ shows the smoothed data, with a running average window of $60 \mathrm{~s}$ in time and $30^{\circ}$ in pitch angle. The smoothed data shows that the $0^{\circ}$ and $180^{\circ}$ electrons are counterstreaming inside the current sheet, consistent with closed magnetic field lines with both ends on the Sun. This is consistent with PSP crossing a sunwarddirected exhaust (Gosling et al. 2006; Lavraud et al. 2009).
The right column of Fig. 6 shows a complete crossing of the HCS on 2018 November 23 during E1. This HCS was previously reported by Phan et al. (2020) but without the discussion of its magnetic topology. Figure $6 \mathrm{~h}$ shows that both $B_{\mathrm{R}}$ and $B_{\mathrm{T}}$ reversed polarity and the magnetic shear across the HCS was $168^{\circ}$. Plasma jetting in the $\mathrm{R}$ and $\mathrm{T}$ directions are seen in the current sheet, but $V_{\mathrm{R}}$ shows an enhancement (relative to the ambient solar wind flow) in this case. The antisunward radial jet indicates that the X-line was sunward of PSP. This event was detected at $107 R_{\mathrm{S}}$, where the strahl electron flux intensity was quite low. Both the raw data and the smoothed data show evidence for dropouts of $0^{\circ}$ and $180^{\circ}$ strahl electrons inside the current sheet, consistent with field lines being disconnected from the Sun in this antisunward exhaust.

The current sheet widths of the 2 HCS discussed in this section are given in Table 1 . They are both of the order of $1000 \mathrm{~d}_{\mathrm{i}}$. The estimated distance from PSP to the X-line, assuming a canonical reconnection rate of 0.1 , is $0.18 R_{\mathrm{S}}$ for E4 HCS 1 , and $1.1 R_{\mathrm{S}}$ for the E1 HCS.

\section{Encounter 5}

\subsection{Overview}

Figure 7 shows that PSP encountered the HCS a number of times during encounter 5 . There are clear HCS crossings with largescale $B_{\mathrm{R}}$ reversals (panel c) and concurrent flipping between $0^{\circ}$ and $180^{\circ}$ strahl electron fluxes across the HCS (panel d). On closer inspections, however, the transitions across the HCS were more complicated than HCS seen on E4: Some E5 full HCS 


\section{Encounter 5}
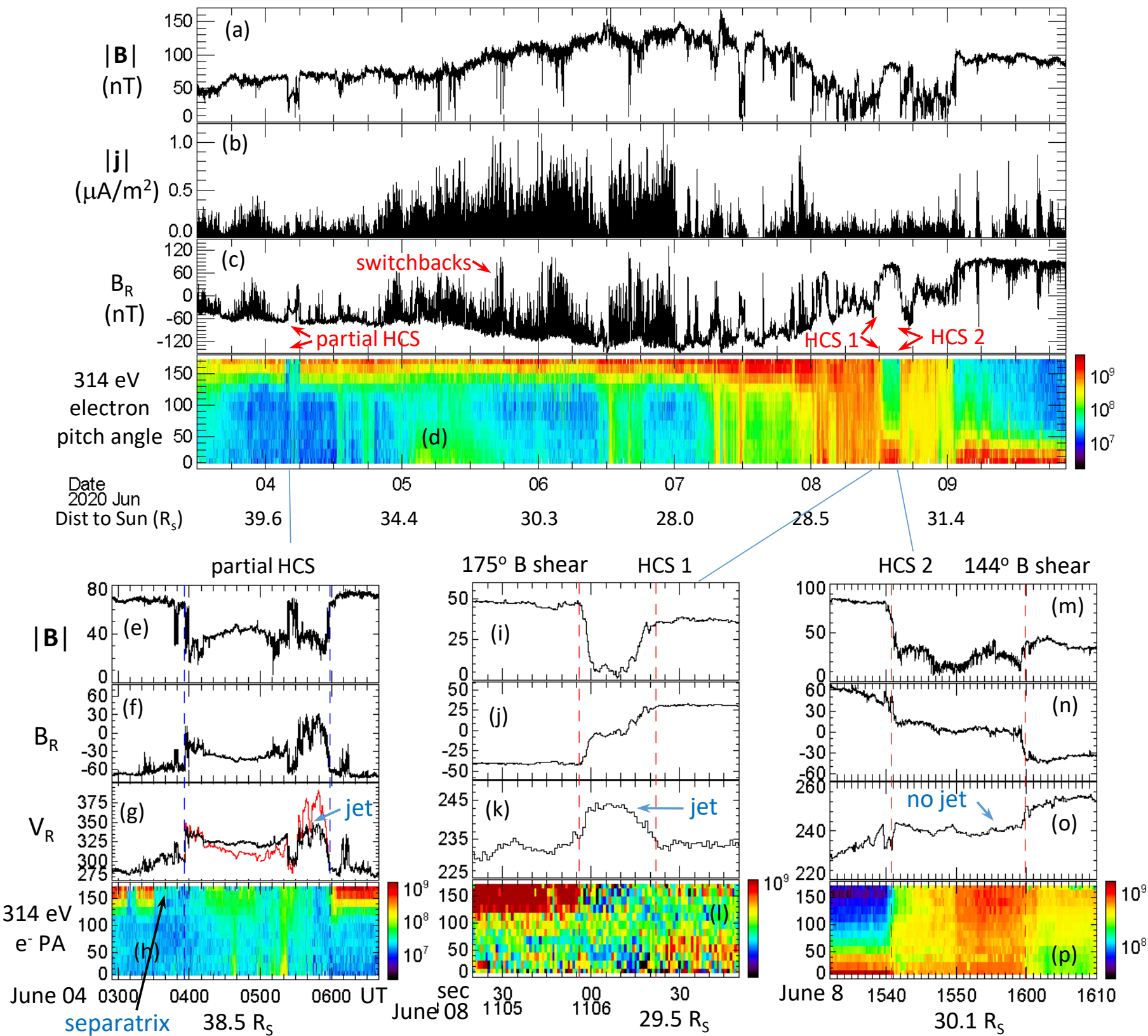

Fig. 7. Panels $a-d$ : overview of encounter 5. Panels $e-h$ : partial crossing of an antisunward exhaust showing magnetic disconnection. Panels $i-l$ : full crossing of an antisunward exhaust showing magnetic disconnection. Panels $m-p$ : full crossing of a HCS showing no reconnection jets, but counterstreaming electrons in the current sheet suggestive of closed field lines. The parameters in panels $a-d$ are the same as in Fig. 1. Panels $e, i$, $m$ : magnitude of the magnetic field. Panels $f, j, n$ : radial components of the magnetic field. Panels $g, k, o$ : radial component of the proton velocity. Panels $h, l, p$ : pitch angle distributions of strahl electrons. The proton moments are from the SWEAP/SPAN-ion measurements and in the Sun's frame. Panel $g$ : the red traces are the Walén predicted $V_{\mathrm{R}}$.

crossings were more drawn out, with strahl electron fluxes not reaching their asymptotic level for a while before or after the main HCS crossings. Nevertheless, we have been able to identify what we believe to be the main portions of the HCS transitions in E5, labeled 'HCS 1' and 'HCS 2' in Fig. 7c. HCS 1 showed evidence for reconnection jets, while HCS 2 did not. We also discuss what appears to be an extended partial HCS crossing that displays roughly Alfvenic accelerated flows. There were other full crossings of the HCS on 2020 June 7 and June 8 (not shown), but their $B_{\mathrm{R}}$ transitions were rather complicated (highly nonmonotonic). Although those crossings show possible evidence for sunward-directed jets $\left(\Delta V_{\mathrm{R}}<0\right)$ and counterstreaming strahl electrons (consistent with closed field lines), the complicated nature of the current sheet crossings makes the identification of reconnection or non-reconnection ambiguous.

\subsection{E5, HCS 1: anti-sunward exhaust and magnetic disconnection}

Figures 7i-1 show a zoom-in of the current sheet crossing labeled 'HCS 1' in Fig. 7c. The current sheet was bifurcated, with sharp changes in $B_{\mathrm{R}}$ at the two edges of the current sheet and a plateau in the middle (Fig. 7j). The magnetic shear across the current sheet was $175^{\circ}$, and $|\mathbf{B}|$ shows a deep minimum (Fig. 7i). The 
$175^{\circ}$ rotation across the current sheet suggests that it is a full crossing of the HCS. A noted feature is that the strahl electron fluxes on the 2 sides of the HCS were very different, with $0^{\circ}$ flux intensity on the trailing edge much lower than the $180^{\circ}$ fluxes on the leading edge. Such a feature may be related to the way the HCS was generated near the Sun (e.g., Crooker et al. 2004; Huang et al. 2016).

A positive $\Delta V_{\mathrm{R}}$ jet was detected in the current sheet, indicating that PSP was in an antisunward-directed exhaust, with the X-line located sunward of PSP. The observed depletion of strahl electrons in the exhaust indicates magnetic disconnection from the Sun. With a crossing duration of $26 \mathrm{~s}$ (width of $\sim 1740 \mathrm{~km}$, or $\sim 240 d_{\mathrm{i}}$ ), this is the thinnest full HCS encountered by PSP during its first five orbits. The estimated distance to the $\mathrm{X}$-line is $9 \times 10^{3} \mathrm{~km}\left(1200 d_{\mathrm{i}}\right)$, or $\sim 0.01 R_{\mathrm{S}}$ (Table 1$)$. The durations of the sharp $\left(B_{\mathrm{R}}\right)$ exhaust boundary crossings were $2.5 \mathrm{~s}$ on the left and $6 \mathrm{~s}$ on the right, which translate to $168 \mathrm{~km}\left(24 d_{\mathrm{i}}\right)$ and $400 \mathrm{~km}$ $\left(57 d_{\mathrm{i}}\right)$, respectively.

\subsection{E5, HCS 2: no reconnection jets, but possibly closed field lines}

Figures 7m-q show a zoom-in of HCS 2 marked in Fig. 7c. The current sheet was bifurcated (Fig. 7n), $|\mathbf{B}|$ showed a deep minimum inside the current sheet (Fig. $7 \mathrm{~m}$ ). The magnetic shear across the current sheet was $144^{\circ}$. Figure $7 p$ shows a signature of counterstreaming strahl electrons inside the current sheet suggestive of closed field lines. These magnetic field and electron characteristics are similar to the reconnection exhausts observed in HCS 1 and 2 in E4. However, there is no evidence for reconnection jets inside this current sheet. The flows in the current sheet were simply Alfvénic, with $\delta \mathrm{B}$ and $\delta \mathrm{V}$ being anti-correlated at both edges of the current sheet.

A possible uncertainty with this current sheet is whether the $144^{\circ}$ field rotation and the highly asymmetric $|\mathbf{B}|$ on the two sides of the current sheet (Fig. $7 \mathrm{~m}$ ) signify that this is not a complete crossing of the HCS. However, $144^{\circ}$ shear represents $80 \%$ of a maximum possible rotation of $180^{\circ}$. Thus the Alfvénic nature of the plasma at both edges of the current sheet strongly suggests a lack of reconnection exhaust. On the other hand, the possible closed field topology suggests that reconnection may have happened earlier, but is no longer active.

\subsection{E5, long-duration partial crossing: anti-sunward exhaust and disconnection}

Figures $7 \mathrm{e}-\mathrm{h}$ show a long $(\sim 2-\mathrm{h})$ interval that we interpret as a partial crossing of the HCS. During the interval between the two vertical dashed lines, $|\mathbf{B}|$ was depressed and $B_{\mathrm{R}}$ showed an excursion away from the negative baseline seen outside the current sheet. In that interval, $V_{\mathrm{R}}$ was positively enhanced (Fig. $7 \mathrm{~g}$ ), and is in qualitative agreement with the Walén prediction (the red trace in Fig. $7 \mathrm{~g}$ ), suggesting that PSP was in an antisunward exhaust, and that reconnection was continuously active for at least two hours. The complete dropout of strahl electron fluxes in the current sheet is consistent with the expected magnetic disconnection from the Sun due to reconnection at a site sunward of PSP. During this interval the net electron heat flux observed by PSP also falls to nearly zero, supporting the identification of a disconnection event (Halekas et al. 2021). The estimated minimum radial dimension of the 2-h structure, given in Table 1, is between 0.5 and $3.5 R_{\mathrm{S}}$, depending on the assumption on the motion of the structure (described in Sect. 5.5).

\section{Discussions}

During three of the first five orbits around the Sun, PSP has provided unprecedented detailed plasma and field observations within and around the near-Sun HCS, at heliocentric distances of 29.5-107 $R_{\mathrm{S}}$. These observations have revealed HCS reconnection properties that are significantly different from those seen at 1 AU. We now summarize and discuss the implications of the PSP observations for (1) the common occurrence of reconnection in the HCS near the Sun, (2) magnetic topology of the reconnecting HCS, and (3) the large-scale structures of the reconnecting HCS.

\subsection{Prevalence of reconnection in the HCS close to the Sun}

At $1 \mathrm{AU}$, reconnection is rarely seen in the HCS (Gosling et al. 2005b). In contrast, during orbits 1,4 , and 5, five out of six welldefined complete HCS crossings by PSP detected reconnection exhaust signatures. In addition, reconnection exhaust jet signatures were detected throughout some of the longest extended (2-3 h in duration) partial HCS crossings by PSP so far. These findings suggest that reconnection is almost always active in the HCS close to the Sun, at least in the solar radial distances of $29.5-107 R_{\mathrm{S}}$ covered in this study. The finding of the prevalence of reconnection in the HCS is somewhat surprising considering the large scale sizes of the HCS. It is also in stark contrast with the recent finding of the absence of reconnection in most of the smaller-scale and much more intense current sheets encountered by PSP near perihelia, many of which are associated with switchbacks (e.g., Figs. 1b and 7b; Phan et al. 2020).

The onset of collisionless reconnection requires current layer thicknesses on the order of the ion inertial length scale (Sanny et al. 1994; Birn et al. 2001; Runov et al. 2008), which is $\sim 10 \mathrm{~km}$ for densities relevant to the present PSP observations. However, there is no evidence that the extended HCS has an intrinsic scale of that order. Thus, the observation that reconnection frequently occurs in the HCS is a surprise. There are two obvious potential explanations for the prevalence of reconnection. The first is that there is local compression of the current sheet due to largescale forcing associated with, for example, the nonuniformity of the solar wind outflow. The second is that the generation of the HCS at the tip of the helmet streamers is nonsteady and results in the generation of reconnecting $\mathrm{X}$-lines that remain active as they propagate outward in the solar wind (Wiegelmann \& Büchner 2000). An active reconnection site would maintain a local thin current sheet as long as there is a continuous supply of free magnetic energy for reconnection to tap into (Shay et al. 2007). Future observations by PSP even closer to the Sun may be able to distinguish between these two scenarios.

\subsection{HCS magnetic topology}

Evidence for the formation of closed magnetic field (e.g., Gosling et al. 2006; Lavraud et al. 2009) and magnetic disconnection from the Sun (e.g., Gosling et al. 2005b) due to reconnection have previously been reported in HCS at 1 AU. PSP observations have added to that knowledge by finding clear correlation between the direction of radial exhaust outflows and the topology of the magnetic field lines inferred from the behavior of the strahl electrons within the current sheet. In the sunward-directed exhausts, PSP detected counterstreaming strahl electrons inside the HCS, indicating that HCS reconnection resulted in the formation of closed magnetic field lines with both ends connected to the Sun. In the antisunward exhausts, 
PSP typically observed dropouts of strahl electrons, consistent with the reconnected HCS field lines being disconnected from the Sun. Magnetic disconnection signatures in HCSs associated with reconnection are similar to recent PSP studies by Szabo et al. (2020) and Lavraud et al. (2020).

So far, we have found two clear examples of sunwarddirected exhaust during full HCS crossings. In both cases, counterstreaming strahl electrons indicative of closed field lines were seen. An interesting question is whether this implies that there were no additional X-lines sunward of PSP for those events, given that reconnection with dominant X-line(s) sunward of the PSP location would have resulted in magnetic disconnection from the Sun. However, one can envision scenarios in which additional X-lines sunward of PSP can happen and still show no magnetic disconnection signatures at PSP. For example, if there is a sequence of magnetic islands (generated by secondary reconnection) convecting sunward (in the frame of the solar wind) by the flow produced by the dominant X-line near PSP, such islands would not eliminate strahl electrons in a measured sunward exhaust flow. Note that we have also seen (not shown) examples of seemingly partial crossings of sunward-directed exhausts with strahl electron flux dropouts, which could indicate the presence of dominant X-line(s) closer to the Sun in those cases.

PSP also observed evidence of the separatrix layers outside some HCS exhausts. However, the separatrix layers are not always present, or are seen only on one side of some exhausts. We currently do not have a firm understanding of the variability of the appearance of the separatrix layer. Because the separatrix electrons are very fast, the dynamic nature of the separatrix could point to the intermittency of the reconnection process at the reconnection site (Lavraud et al. 2009), or to the open/closed nature of the exhaust boundaries (Sasunov et al. 2012; Shepherd et al. 2017). Another explanation for the various kinds of separatrix-like behavior is the different ways the HCS may be generated near the Sun (e.g., Huang et al. 2016).

\subsection{Large-scale structures of the reconnecting HCS}

The occurrence of multiple and some extremely long partial crossings of the HCS exhibiting exhaust signatures indicates that the reconnecting HCS is not a narrow and linearly expanding exhaust. These partial crossings suggest the presence of large $\left(\mathrm{R}_{\mathrm{S}}\right.$-scale) blobs as depicted in Fig. 5j. Such bulges along the HCS could be flux ropes generated by multiple Xline (e.g., Sanchez-Diaz et al. 2019; Lavraud et al. 2020; Réville et al. 2020). However, in the events described in the previous sections, the exhaust radial jets throughout the long duration exhausts, as well as in shorter consecutive partial crossings, were unidirectional. Thus, the blobs were likely not convecting magnetic islands/flux ropes flanked by active X-lines, which should have produced alternating sunward and antisunward jet signatures. One cannot, however, exclude the possibility that the blobs were created earlier by multiple reconnection, but their associated X-lines ceased to be active, except for a dominant one which sweeps all the others downstream. Such a scenario is often seen in kinetic simulations (e.g., Shay et al. 1999; Daughton \& Karimabadi 2007), albeit on much smaller scales. Another way to generate blobs in the exhaust with a single X-line is to modulate the reconnection rate (e.g., Scholer 1988; Phan et al. 2004).

The presence of blobs along the HCS has previously been seen in remote-sensing observations (e.g., Rouillard et al. 2010a,b; Sanchez-Diaz et al. 2017; Howard et al. 2019). It is presently unclear whether the $\mathrm{R}_{\mathrm{S}}$-scale blobs that we deduced from in-situ observations are similar to those seen in the remotesensing images.

Another observed property of the HCS exhaust is the sharpness of the exhaust boundaries, as evidenced by the abrupt changes of $B_{\mathrm{R}}$ at the edges of the current sheet seen in both complete and partial crossings of the HCS. This provides evidence that the reconnection exhausts are bounded by slow-shock-like structures (e.g., Petschek 1964; Gosling et al. 2005a; Phan et al. 2006, 2020; Mistry et al. 2015). It also provides further support for the interpretation that the 'partial' crossings are crossings into the reconnecting HCS. That such sharp boundaries can persist over large distances from the X-line is remarkable, and they would be consistent with the MHD picture of large-scale reconnection (Petschek 1964). The sharp HCS boundaries detected by PSP during full crossings are well resolved by the PSP measurements, and are a few tens of $\mathrm{d}_{\mathrm{i}}$ thick.

In conclusion, PSP has provided remarkable observations of the HCS through its first five orbits. The occurrence rate of reconnection in HCS at 29.5-107 $R_{\mathrm{S}}$ appears to be much higher than at $1 \mathrm{AU}$, and HCS reconnection seems to be continuously active at these radial distances. It remains to be seen how the HCS reconnection properties change as PSP gets even closer to the Sun.

Acknowledgements. We are grateful for the dedicated efforts of the entire Parker Solar Probe team. We thank Chris Chaston, Bill Daughton, Mark Linton, and Yuan-Kuen Ko for helpful discussions. We acknowledge NASA FIELDS contract NNN06AA01C, NASA SWEAP contract NNN06AA01C, NASA grants 80NSSC20K1781, 80NSSC20K0627, and NNX17AI25G, and UK STFC grant ST/S000364/1. Work at IRAP and LAB was supported by CNRS and CNES. We wish to acknowledge support from the International Space Science Institute, Bern.

\section{References}

Angelopoulos, V., Kennel, C. F., Coroniti, F. V., et al. 1994, J. Geophys. Res., 99, 21257

Badman, S. T., Bale, S. D., Rouillard, A. P., et al. 2021, A\&A, 650, A18 (PSP SI)

Bale, S. D., Goetz, K., Harvey, P. R., et al. 2016, Space Sci. Rev., 204, 49

Bale, S. D., Badman, S. T., Bonnell, J. W., et al. 2019, Nature, 576, 237

Birn, J., Drake, J. F., Shay, M. A., et al. 2001, J. Geophys. Res., 106, 3715

Case, A. W., Kasper, J. C., Stevens, M. L., et al. 2020, ApJS, 246, 43

Cattell, C. A., Short, B., Breneman, A. W., \& Grul, P. 2020, ApJ, 897, 126

Cattell, C., Glesener, L., Leiran, B., et al. 2021, A\&A, 650, A6 (PSP SI)

Chen, C. H. K., Chandran, B. D. G., Woodham, L. D., et al. 2021, A\&A, 650, L3 (PSP SI)

Crooker, N. U., Larson, D. E., Kahler, S. W., Lamassa, S. M., \& Spence, H. E. 2003, Geophys. Res. Lett., 30, 1619

Crooker, N. U., Huang, C.-L., Lamassa, S. M., et al. 2004, J. Geophys. Res. Space Phys., 109, A3

Daughton, W., \& Karimabadi, H. 2007, Phys. Plasmas, 14, 072303

Davis, M. S., Phan, T. D., Gosling, J. T., \& Skoug, R. M. 2006, Geophys. Res. Lett., 33, L19102

Doss, C. E., Cassak, P. A., \& Swisdak, M. 2016, Phys. Plasmas, 23, 082107

Drake, J. F., Swisdak, M., Phan, T. D., et al. 2009, J. Geophys. Res., 114, 5111

Eriksson, S., Gosling, J. T., Phan, T. D., et al. 2009, J. Geophys. Res. Space Phys., 114, A07103

Fox, N. J., \& McComas, D. J. 2016, Space Sci. Rev., 204, 1

Frey, H. U., Phan, T. D., Fuselier, S. A., \& Mende, S. B. 2003, Nature, 426, 533

Gosling, J. T. 2007, ApJ , 671, L73

Gosling, J. T., Skoug, R. M., McComas, D. J., \& Smith, C. W. 2005a, J. Geophys. Res., 110, 1107

Gosling, J. T., Skoug, R. M., McComas, D. J., \& Smith, C. W. 2005b, Geophys. Res. Lett., 32, L05105

Gosling, J. T., McComas, D. J., Skoug, R. M., \& Smith, C. W. 2006, Geophys. Res. Lett., 33, L17102

Gosling, J. T., Phan, T. D., Lin, R. P., \& Szabo, A. 2007, Geophys. Res. Lett., 34, L15110

Haggerty, C. C., Shay, M. A., Drake, J. F., Phan, T. D., \& McHugh, C. T. 2015, Geophys. Res. Lett., 42, 9657

Haggerty, C. C., Shay, M. A., Chasapis, A., et al. 2018, Phys Plasmas, 25, 102120 
Halekas, J. S., Whittlesey, P. L., Larson, D. E., et al. 2020, ApJS, 246, 22 Halekas, J. S., Whittlesey, P. L., Larson, D. E., et al. 2021, A\&A, 650, A15 (PSP SI)

Horbury, T. S., Matteini, L., \& Stansby, D. 2018, MNRAS, 478, 1980

Horbury, T. S., Woolley, T., Laker, R., et al. 2020, ApJS, 246, 45

Howard, R. A., Vourlidas, A., Bothmer, V., et al. 2019, Nature, 576, 232

Huang, J., Liu, Y. C. M., Qi, Z., et al. 2016, J. Geophys. Res. Space Phys., 121, 10,768

Hudson, P. D. 1970, Planet. Space Sci., 18, 1670

Huttunen, K. E. J., Bale, S. D., \& Salem, C. 2008, Ann. Geophys., 26, 2701

Kasper, J. C., Abiad, R., Austin, G., et al. 2016, Space Sci. Rev., 204, 131

Kasper, J. C., Bale, S. D., Belcher, J. W., et al. 2019, Nature, 576, 228

Kronberg, P. P. 2002, Phys. Today, 55, 12.40

La Belle-Hamer, A. L., Otto, A., \& Lee, L. C. 1995, J. Geophys. Res., 100, 875

Lavraud, B., Gosling, J. T., Rouillard, A. P., et al. 2009, Sol. Phys., 256, 379

Lavraud, B., Ruffenach, A., Rouillard, A. P., et al. 2014, J. Geophys. Res. Space Phys., 119, 26

Lavraud, B., Fargette, N., Réville, V., et al. 2020, ApJ, 894, L19

Liu, Y.-H., Drake, J. F., \& Swisdak, M. 2012, Phys. Plasmas, 19, 022110

Livi, R., Larson, D. E., Kasper, J. C., et al. 2020, ApJS, submitted

Mistry, R., Eastwood, J. P., Phan, T. D., \& Hietala, H. 2015, Geophys. Res. Lett., 42, 513

Mistry, R., Eastwood, J. P., Phan, T. D., \& Hietala, H. 2017, J. Geophys. Res. Space Phys., 122, 5895

Øieroset, M., Phan, T. D., Lin, R. P., \& Sonnerup, B. U. Ö. 2000, J. Geophys. Res., 105, 25247

Pagel, C., Crooker, N. U., \& Larson, D. E. 2005, Geophys. Res. Lett., 32, L14105

Parker, E. N. 1983, ApJ, 264, 642

Paschmann, G., Baumjohann, W., Sckopke, N., Papamastorakis, I., \& Carlson, C. W. 1986, J. Geophys. Res., 91, 11099

Paschmann, G., Øieroset, M., \& Phan, T. 2013, Space Sci. Rev., 178, 385

Petschek, H. E. 1964, in AAS/NASA Symposium on the Physics of Solar Flares, ed. W. N. Ness (Washington DC: NASA), 425

Phan, T. D., \& Paschmann, G. 1996, J. Geophys. Res., 101, 7801

Phan, T.-D., Paschmann, G., \& Sonnerup, B. U. Ö. 1996, J. Geophys. Res., 101, 7817

Phan, T., Dunlop, M., Paschmann, G., et al. 2004, Ann. Geophys., 22, 2355

Phan, T. D., Gosling, J. T., Davis, M. S., et al. 2006, Nature, 439, 175

Phan, T. D., Gosling, J. T., \& Davis, M. S. 2009, Geophys. Res. Lett., 36, L09108

Phan, T. D., Gosling, J. T., Paschmann, G., et al. 2010, ApJ, 719, L199
Phan, T. D., Paschmann, G., Gosling, J. T., et al. 2013, Geophys. Res. Lett., 40, 11

Phan, T. D., Drake, J. F., Shay, M. A., et al. 2014, Geophys. Res. Lett., 41, 7002 Phan, T. D., Eastwood, J. P., Shay, M. A., et al. 2018, Nature, 557, 202

Phan, T. D., Bale, S. D., Eastwood, J. P., et al. 2020, ApJS, 246, 34

Priest, E. R. 1984, Washington DC American Geophysical Union Geophysical Monograph Series, 30, 63

Réville, V., Velli, M., Panasenco, O., et al. 2020, ApJS, 246, 24

Rouillard, A. P., Davies, J. A., Lavraud, B., et al. 2010a, J. Geophys. Res. Space Phys., 115, A04103

Rouillard, A. P., Lavraud, B., Davies, J. A., et al. 2010b, J. Geophys. Res. Space Phys., 115, A04104

Ruffenach, A., Lavraud, B., Owens, M. J., et al. 2012, J. Geophys. Res. Space Phys., 117, A09101

Ruffenach, A., Lavraud, B., Farrugia, C. J., et al. 2015, J. Geophys. Res. Space Phys., 120, 43

Runov, A., Baumjohann, W., Nakamura, R., et al. 2008, J. Geophys. Res. Space Phys., 113, A07S27

Sanchez-Diaz, E., Rouillard, A. P., Davies, J. A., et al. 2017, ApJ, 835, L7

Sanchez-Diaz, E., Rouillard, A. P., Lavraud, B., Kilpua, E., \& Davies, J. A. 2019, ApJ, 882, 51

Sanny, J., McPherron, R. L., Russell, C. T., et al. 1994, J. Geophys. Res., 99, 5805

Sasunov, Y. L., Semenov, V. S., Heyn, M. F., Kubyshkin, I. V., \& Biernat, H. K. 2012, Geophys. Res. Lett., 39, L06104

Scholer, M. 1988, Geophys. Res. Lett., 15, 291

Shay, M. A., Drake, J. F., Rogers, B. N., \& Denton, R. E. 1999, Geophys. Res. Lett., 26, 2163

Shay, M. A., Drake, J. F., \& Swisdak, M. 2007, Phys. Rev. Lett., 99, 155002

Shay, M. A., Haggerty, C. C., Phan, T. D., et al. 2014, Phys. Plasmas, 21, 122902

Shepherd, L. S., Cassak, P. A., Drake, J. F., et al. 2017, ApJ, 848, 90

Sonnerup, B. U. O., \& Cahill, L. J., J. 1967, J. Geophys. Res., 72, 171

Sonnerup, B. U. O., Paschmann, G., Papamastorakis, I., et al. 1981, J. Geophys. Res., 86, 10049

Swisdak, M., Rogers, B. N., Drake, J. F., \& Shay, M. A. 2003, J. Geophys. Res., 108,1218

Swisdak, M., Opher, M., Drake, J. F., \& Alouani Bibi, F. 2010, ApJ, 710, 1769

Szabo, A., Larson, D., Whittlesey, P. L., et al. 2020, ApJS, 246, 47

Whittlesey, P. L., Larson, D. E., Kasper, J. C., et al. 2020, ApJS, 246, 74

Wiegelmann, T., \& Büchner, J. 2000, Nonlinear Process. in Geophys., 7, 141

Yamada, M., Kulsrud, R., \& Ji, H. 2010, Rev. Mod. Phys., 82, 603 